\title{
Gradhiva
}

GRADHIV

Revue d'anthropologie et d'histoire des arts

$7 \mid 2008$

Le possédé spectaculaire

Jérôme Souty, Pierre Fatumbi Verger. Du regard détaché à la connaissance initiatique

Paris, Maisonneuve et Larose, 2007, 517 p.

\section{Erwan Dianteill}

\section{OpenEdition}

\section{Journals}

Édition électronique

URL : http://journals.openedition.org/gradhiva/1131

DOI : $10.4000 /$ gradhiva. 1131

ISSN : 1760-849X

\section{Éditeur}

Musée du quai Branly Jacques Chirac

Édition imprimée

Date de publication : 15 mai 2008

Pagination : 159-160

ISBN : 978-2-915133-86-8

ISSN : 0764-8928

Référence électronique

Erwan Dianteill, « Jérôme Souty, Pierre Fatumbi Verger. Du regard détaché à la connaissance initiatique », Gradhiva [En ligne], 7 | 2008, mis en ligne le 10 décembre 2008, consulté le 21 septembre 2020. URL http://journals.openedition.org/gradhiva/1131 ; DOI : https://doi.org/10.4000/gradhiva.1131

Ce document a été généré automatiquement le 21 septembre 2020.

(C) musée du quai Branly 


\section{Jérôme Souty, Pierre Fatumbi Verger. Du regard détaché à la connaissance initiatique}

Paris, Maisonneuve et Larose, 2007, 517 p.

\section{Erwan Dianteill}

\section{RÉFÉRENCE}

Jérôme Souty, Pierre Fatumbi Verger. Du regard détaché à la connaissance initiatique, préface de Jean-Paul Colleyn. Paris, Maisonneuve et Larose, 2007, 517 p.

1 En 2002, une première biographie de Pierre Verger était publiée au Brésil. Signée par Jean-Pierre Le Bouler et intitulée Pierre Fatumbi Verger, um homem livre (Salvador de Bahia, Fundação Pierre Verger), cet ouvrage très bien documenté donnait un compte rendu factuel de la vie de Verger, et il reste aujourd'hui très utile. Le livre de Jérôme Souty, préfacé par Jean-Paul Colleyn qui en montre toute l'originalité, est tout à fait différent, non que l'aspect biographique en soit absent, mais l'interrogation porte avant tout ici sur la nature et la portée d'une œuvre protéiforme : «La méthode originale et non conventionnelle que Verger a mise en pratique (comme photographe puis comme ethnographe), écrit Souty, est indissociable de son œuvre et de son parcours de vie » (p. 23). Quel enseignement peut-on tirer alors de l'itinéraire de vie du photographeethnographe? En quoi sa démarche interroge-t-elle les fondements de l'anthropologie? Souty tente de répondre à ces questions par un va-et-vient constant entre éléments biographiques et analyse des œuvres scientifiques et artistiques de Verger. Il nous convie ainsi à examiner ce que peut être une « œuvre-vie ».

D'abord photographe (l'ouvrage de Souty est illustré d'une iconographie d'une grande beauté, qui permet de mesurer son talent dans ce domaine), Verger ne se découvre ethnographe qu'au milieu des années 1930. Sa méthode est plutôt une "antiméthode ", en tout cas une "méthode par défaut " selon les mots de Souty: Verger a 
collecté une somme d'informations sans les rechercher activement ou volontairement. Né en 1902, il est resté en marge du système académique jusqu'à 60 ans (âge auquel il devient chercheur statutaire au CNRS), ce qui lui a donné une absolue liberté de travail. De ce point de vue, il a une trajectoire proche de celle de Lydia Cabrera, avec qui il entretient des relations amicales. Celle-ci compose en effet dans les années 1950 une somme sur les religions afro-cubaines - El Monte ${ }^{1}$ - sans aucun soutien universitaire. Leur façon de faire est d'ailleurs très comparable, l'un et l'autre pratiquant une sorte de cordialité méthodologique, renonçant au scientisme et à la posture de supériorité culturelle assez fréquente chez les ethnologues de leur temps.

Photographe-ethnographe, Verger est aussi un chercheur initié dans les religions dont il fut un spécialiste. En premier lieu, Souty montre que ces initiations furent précédées d'un long processus de rupture avec son milieu d'origine, sa famille, son pays. Après avoir perdu ses parents les plus proches, il adopte un style de vie «bohème » à Paris et, en 1932, se donne dix ans à vivre, après quoi il décide qu'il se suicidera (résolution qu'il ne mettra pas à exécution). Suivent des années de voyage dans le monde entier, l'Europe du Sud, Tahiti, le Pérou, le Mexique, pendant lesquelles Verger tire un grand nombre de clichés publiés dans la presse. Il finit par s'installer à Salvador de Bahia, où il lui semble que sa couleur de peau n'est pas un obstacle à son intégration. En second lieu, à partir de 1948, Verger est initié dans le culte des orisha. Les premières initiations se font à Salvador en 1948, où il reçoit le collier de Shango, dieu de la foudre, puis au Dahomey et au Nigeria. Autre moment important, en 1953, Verger devient babalao, interprète légitime de l'oracle d'Ifa, à Ketu, non loin de Porto-Novo. Il change de nom et devient Fatumbi, « celui-qui est né d'Ifa ». Il apprend alors la signification des oracles et la mythologie qui leur est associée. En 1966, il a collecté un corpus d'environ deux mille histoires distinctes (p. 337) et devient en outre un spécialiste de la pharmacopée associée à ces signes. Son dernier livre, intitulé Ewé2, constitue un bilan de ses recherches en ethnobotanique. Verger fut aussi initié dans plusieurs sociétés secrètes (Egun, Oro...). Tout compte fait, il écrivit peu sur l'ensemble de ses expériences initiatiques. Et il ne publia pas le grand ouvrage attendu sur les mythes d'Ifa, bien qu'il fût apparemment rédigé. Lorsqu'on lit Souty, l'initiation semble chez Verger le résultat naturel d'une longue familiarité avec la culture yoruba et brésilienne plutôt qu'une entreprise de découverte d'une vérité cachée. Sur ce sujet, le dernier chapitre de l'ouvrage intitulé «La connaissance initiatique » est particulièrement stimulant. Souty y montre bien que, pour Verger, «l'initiation [...] c'est vivre avec les gens » (p. 454), processus dont la charge mystique est marginale, sinon complètement absente. L'effet le plus saillant de l'initiation est alors qu'elle peut déstabiliser l'ethnocentrisme et permettre une connaissance recentrée.

4 Souty n'est pas le premier à s'intéresser au personnage hors du commun que fut Pierre Verger, mais son livre est néanmoins exceptionnel par son ampleur, la qualité de l'iconographie présentée et surtout l'intelligence de l'articulation entre œuvre scientifique, œuvre artistique et biographie. À l'heure où certains balaient d'un revers de main l'histoire de la discipline au nom de la science " en marche ", cet ouvrage est exemplaire de ce qu'une biographie d'ethnographe peut apporter à l'anthropologue d'aujourd'hui. 


\section{NOTES}

1. Lydia Cabrera, La Forêt et les Dieux. Religions afro-cubaines et médecine sacrée à Cuba. Paris, JeanMichel Place ("Cahiers de Gradhiva »), 2002.

2. Ewé, o uso de plantas na sociedade ioruba. São Paulo, Companhia das Letras, 1995.

\section{AUTEURS}

ERWAN DIANTEILL

erwan.dianteill@quaibranly.fr 